\title{
Bearing estimation using a perturbed linear array*
}

\author{
Melvin J. Hinich \\ Department of Economics, Virginia Polytechnic Institute and State University, Blacksburg, Virginia 24061 \\ (Received 30 April 1976; revised 2 January 1977)

\begin{abstract}
A linear hydrophone array which is towed in the ocean is subject to snakelike bending. If the array is processed as if it was truly linear, the author has shown that the bending causes a deflection of the measured bearing of a fixed source from its true bearing relative to the array. This deflection results from patterned perturbations in the true sensor positions along the nominal array axis. As the perturbation pattern changes with the flexing of the array, the source appears to move around. A probability model of the perturbations is used in order to develop a theoretical solution to the question of how the space-time information gathered by the array is best used to measure source bearing. The method which is used to reduce the bending perturbation deflection of the bearing is to group the sensors into adjacent subarrays, process these arrays over short time slices, average the subarray bearings for each time period, and then to average the average over time. This averaging method significantly improves the bearing accuracy of the
\end{abstract} \\ source when the array is bent according to the model.
}

PACS numbers: $43.60 . \mathrm{Cg}, 43.60 . \mathrm{Gk}, 43.30 . \mathrm{Vh}$

\section{FREQUENCY-WAVE-NUMBER BEARING ESTIMATION}

Beamforming is the most commonly used signal processing technique for measuring the bearing of a farfield source using a linear array of sensors. If the noise is Gaussian and incoherent, then Levin ${ }^{1}$ has shown that the maximum likelihood estimator of the direction of arrival of a plane wave is the beam angle which maximizes the total received energy in the $1 / T \mathrm{~Hz}$ bands in which the signal has energy. Levin's analysis is rigorously extended to a more general plane-wave model by Hinich and Shaman. ${ }^{2}$ Their frequency-wave-number approach is exposited by Clay, Hinich, and Shaman. ${ }^{3}$ Other approaches to array processing using different assumptions about the signal and noise field are given by Bryn, ${ }^{4}$ Burg, ${ }^{5}$ Capon et al.,${ }^{6}$ Ligett, ${ }^{7}$ Hahn, ${ }^{8}$ and Carter and Knapp ${ }^{9}$ (for additional references see Refs. 1 and 3).

In order to simplify the exposition, suppose that the signal detected by a sensor located at $x$ at time $t$ is a narrow-band plane-wave signal plus noise, denoted in complex variable notation

$$
p(t, x)=A \exp \left\{i \omega_{0}[t-(x / c) \cos \theta]\right\}+n(t, x),
$$

where $A$ is a complex amplitude which is slowly varying, $c$ is the phase velocity of propagation, $\omega_{0}$ is the angular frequency, and $\theta$ is the direction of propagation with respect to the array axis. The expected value of the noise $n(x, t)$ is zero. Suppose that each sensor is simultaneously sampled for a $T$-sec period using a fixed sampling rate.

A mathematical equivalent of delay-and-sum beamforming, called frequency-wave-number analysis, uses the time-space Fourier transform of the signal, namely,

$$
P(\omega, \kappa)=\frac{1}{M T} \sum_{m=0}^{M-1} \sum_{t_{j}=0}^{T} p\left(t_{j}, x_{m}\right) \exp \left[i\left(\kappa x_{m}-\omega t_{j}\right)\right],
$$

where $x_{m}$ is the position of the $m$ th sensor in an $M$-element array and $t_{j}$ is the $j$ th observation of the sensor outputs. Counting from zero is used to simplify Fourier-transform mathematics. For the $\omega_{0}$ frequency component, the maximum likelihood estimator of $\theta$ is

$$
\hat{\theta}=\arccos \left(c \omega_{0}^{-1} \hat{\kappa}\right),
$$

where $\hat{\kappa}$ is the wave number which maximizes the frequency-wave-number power spectrum

$$
S\left(\omega_{0}, \kappa\right)=M\left|P\left(\omega_{0}, \kappa\right)\right|^{2} .
$$

The $M$ is used in (3) to conform with the usual definition of $S$ in the literature.

There are three main reasons for working in the frequency-wave-number domain. First, it is faster to compute, especially if the sensors are equally spaced, i.e., $x_{m}=m d$, where $d$ is the spacing distance and $-\pi / d$ $\leqslant \kappa<\pi / d$. Second, the use of shading weights to control $\omega$ and $\kappa$ side lobes is made computationally easier, and third, the orthogonal properties of the discrete Fourier coefficients $P\left(\omega, \kappa_{m}\right)$ makes it easier to analyze the noise effects. For example, if the noise field is coherent or nonwhite, the spectrum $S(\omega, \kappa)$ can be simply adjusted by dividing by the square root of the noise $\omega-\kappa$ spectrum if it is known. ${ }^{3}$ Such an adjustment "prewhitens" the spectrum if $M$ or $T$ is not too small, and the maximum likelihood properties of $\hat{\theta}$ still hold. Moreover, the linearity of the Fourier transform results in a Gaussian distribution of the Fourier coefficients, provided that the noise has finite higher moments.

The maximum likelihood estimator has minimum mean-square error (mse) when $\rho$, the energy signal-tonoise ratio in the $1 / T \mathrm{~Hz}$ band centered at $\omega_{0}$, or $M$ is large. ${ }^{3,10}$ The mean-square error for large $M$ or $\rho$ is

$$
\mathrm{mse}(\hat{\theta}) \simeq \frac{3}{2 M W^{2} \rho(\pi \sin \theta)^{2}}, \quad \theta \neq 0, \pi
$$

where $W$ is the aperture of the array as measured by the number of wavelengths $\lambda_{0}=2 \pi c / \omega_{0}$, i.e., $W=L / \lambda_{0}$ for an array of length $L$.

This result is $\sqrt{3}$ times the mse approximation given by MacDonald and Schultheiss. ${ }^{11}$ They show that the Cramer-Rao lower bound for the variance of an unbiased estimator of $\theta$ is minimized by lumping the sensors into 
two clusters, one at each end of the array. This result holds for a Gaussian noise field which is spatially incoherent across the array. The mean-square error of the maximum likelihood estimator attains the CramerRao bound in the limit as the sample size ( $M$ in this case) goes to infinity, ${ }^{12,13}$ provided that the array covariance matrix of the noise remains nonsingular in the limit. This will not be the case for a "thick" dipolar cluster array if the noise field has some spatial coherence. The MacDonald-Schultheiss analysis holds in the limit for a two-element interferometer ${ }^{14}$ as the noise variance goes to zero. ${ }^{15}$ The approximation given by Eq. (4) holds for a high-gain array whose sensors are spread out (see Clay and Hinich ${ }^{16}$ ).

When the array aperture is small or the post-filtering signal-to-noise is small, the measured bearing can be very bad. If, however, the source continues to radiate energy whose waves are coherent at the array, the sampling interval can be made sufficiently long in order to have a $\rho$ which is large enough for (4) to hold. On the other hand, an alternative sampling procedure is to keep $T$ fixed and use a sequence of $\hat{\theta}$ estimates to locate the source. This scheme allows the operator to use visual cues obtained from processor output plus other data in order to track to source. This paper uses a stochastic model of sensor locations and the above approximation to analyze space and time sampling tradeoffs when the array is bending over time, extending a previous paper by the author and a co-worker on bent linear arrays. ${ }^{17}$

\section{PERTURBATIONS IN THE ARRAY GEOMETRY}

It is obvious that there is some positional uncertainty for the sensors in a fixed array, but the errors are usually a negligible fraction of the signal wavelengths. Moreover, test sources can be used to reduce the positional uncertainty of a fixed array. ${ }^{18}$ In contrast, a hydrophone array which is towed in the ocean is subject to snakelike bending and stretching, owing to slight variations in the speed and course of the tow ship. If the array has a sinusoidal or damped sinusoidal pattern, Hinich and Rule show that even a $2 \%$ bending amplitude is enough to cause a significant deflection of the measured bearing from the true $\theta$. This deflection results from patterned perturbations in the true sensor positions along the nominal array axis. As the perturbation pattern changes, the source appears to move around.

It is obvious that the computed bearing will have a significant error if the center of mass of the hydrophones shifts from the nominar array axis, as is the case for an array which has an $L$-shaped pattern. It is not so obvious that perturbations in the array geometry which do not significantly alter the center of mass can degrade bearing accuracy.

Hinich and Rule suggest measuring the source bearing by averaging bearings obtained by processing the array as a set of smaller-aperture linear arrays. The method is studied using artificial data. Recognizing the limitations of a nonlinear geometry which requires the use of artificial data, this paper uses a stochastic mod- el of the perturbations in order to develop a theoretical solution to the question of how the time-space information gathered by an array is to be best used to measure a fixed-source bearing.

Assume, for simplicity, that the $m$ th sensor position is $x_{m}=m d$ if the array is truly linear. When the array is bent, let $u_{m}(t)$ denote the perturbation of the $m$ th sensor's position along the array axis (the $x$ axis) and $y_{m}(t)$ denote the perturbation along the $y$ axis, i.e., at time $t$, the sensor coordinates are $x_{m}(t)=m d+u_{m}(t)$ and $y_{m}(t)$. Although the bending pattern is nonstationary and depends upon the mass and elasticity of the array structure, the observed patterns suggest that each perturbation term is correlated across the array over time. As a statistically convenient model, suppose that for each $m$ and $t, u_{m}(t)$ and $y_{m}(t)$ are zero mean Gaussian random variables with a common variance $\sigma_{u}^{2}$ which is independent of $m$ and $t$. Assume that for each $m, m^{\prime}, t$, and $t^{\prime}$, the covariance between $u_{m}(t)$ and $u_{m^{\prime}}\left(t^{\prime}\right)$ is given by

$$
E u_{m}(t) u_{m^{\prime}}\left(t^{\prime}\right)=\sigma_{u}^{2} \gamma^{\left|m-m^{\prime}\right|} \eta^{\left|t-t^{\prime}\right|},
$$

where the time unit is set equal to the sampling interval (and thus $t$ is an integer), and the correlation coefficients $\gamma$ and $\eta$ are stationary over the sampling period. These correlation coefficients depend, however, on the sensor spacing and the sampling rate because they are dimensionless. In addition, suppose that $y_{m}(t)$ is uncorrelated with $u_{m}(t)$ and has the same covariance structure as that of $u_{m}(t)$. The most unrealistic assumption, in my estimation, is that $u_{m}$ and $y_{m}$ have the same variance, but it greatly simplifies the analysis without destroying the insights about the degradation of bearing accuracy which this stochastic model provides. This simple model also allows us to approximate the gain achieved by subarray processing.

Let $\kappa_{0}=2 \pi / \lambda_{0}$ denote the wave number. When the array is processed as if $x_{m}=m d$ it follows from (1) and (2) that

$$
P\left(\omega_{0}, \kappa\right)=A B\left(\kappa_{0}\right)+N\left(\omega_{0}, \kappa\right),
$$

where

$B\left(\kappa_{0}\right)=(M T)^{-1} \sum_{m=0}^{M-1} \sum_{t=0}^{T} \exp \left\{i \kappa_{0}\left[u_{m}(t) \cos \theta+y_{m}(t) \sin \theta\right]\right\}$,

and $t$ is an integer since the time unit was set equal to the sampling interval. The real and imaginary components of the Fourier coefficient of the noise, $N\left(\omega_{0}, \kappa\right)$, have zero means and the same variance $1 / 2 S_{n}\left(\omega_{0}, \kappa\right)$, where $S_{n}\left(\omega_{0}, \kappa\right)$ denotes the frequency-wave-number power spectrum of the noise in a region about $\left(\omega_{0}, \kappa\right)$ of width $1 / T$ and height $1 / L$, where $L=M d$ is the length of the array. Thus the noise term $N$ is of the order $O\left((M T)^{-1 / 2}\right)$, and the spectrum $S\left(\omega_{0}, \kappa\right)$ from (3) and (6) has a peak at $\kappa=\kappa_{0}$ whose value is approximately $M\left|A B\left(\kappa_{0}\right)\right|^{2}$. The side lobes of the spectrum, which are $5 \%$ of the main lobe, can be reduced by tapering the amplitudes of the end sensors in each subarray.

$$
\begin{aligned}
& \text { The expected value of } B\left(\kappa_{0}\right) \text { is } \\
& \qquad \begin{aligned}
E B\left(\kappa_{0}\right) & =E \exp \left\{i \kappa_{0}\left[u_{m}(t) \cos \theta+y_{m}(t) \sin \theta\right]\right\} \\
& =\exp \left(-1 / 2 \kappa_{0}^{2} \sigma_{u}^{2}\right)
\end{aligned}
\end{aligned}
$$


because $u_{m}(t)$ and $y_{m}(t)$ are (jointly Gaussian) uncorrelated random variables with common variance $\sigma_{u}^{2}$. If the variances were unequal or if the perturbations were correlated, then the expected value of $B\left(\kappa_{0}\right)$ would depend on $\theta$.

The perturbation effect $B\left(\kappa_{0}\right)$ has a variance. The variance of the $m$ th component of the sum (6) is just $1-\exp \left(-\kappa_{0}^{2} \sigma_{u}^{2}\right)$, which is approximately $\left(\kappa_{0} \sigma_{u}\right)^{2}$ if $\sigma_{u} / \lambda_{0}$ is small. The covariance between the $m t$ th and $m^{\prime} t^{\prime}$ th component $\left(m \neq m^{\prime}, t \neq t^{\prime}\right)$ is

$$
\exp \left(-\kappa_{0}^{2} \sigma_{u}^{2}\right)\left[\exp \left(\kappa_{0}^{2} \sigma_{u}^{2} \gamma^{\left|m-m^{\prime}\right|} \eta^{\left|t-t^{\prime}\right|}\right)-1\right]
$$

because $u_{m}(t)-u_{m^{\prime}}\left(t^{\prime}\right)$ is Gaussian with variance $2 \sigma_{u}^{2}(1$ $\left.-\gamma^{\left|m-m^{\prime}\right|} \eta^{\left|t-t^{\prime}\right|}\right)$, and similarly for $y_{m}(t)-y_{m^{\prime}}\left(t^{\prime}\right)$. It then follows from (6) that for small $\sigma_{u} / \lambda_{0}$ and large $M T$,

$$
\begin{aligned}
\operatorname{Var} B \simeq & \left(\kappa_{0} \sigma_{u}\right)^{2}(M T)^{-2} \\
& \times\left(M+2 \sum_{m=1}^{M-1}(M-m) \gamma^{m}\right)\left(T+2 \sum_{t>0}(T-t) \eta^{t}\right) \\
\simeq & \frac{1+\gamma}{1-\gamma} \frac{1+\eta}{1-\eta} \frac{\left(\kappa_{0} \sigma_{u}\right)^{2}}{M T} .
\end{aligned}
$$

When $M T$ is large the variance of $B$ is small, and thus $E|B|^{2} \simeq(E B)^{2}$. To give some numerical examples, suppose that $\sigma_{u}=\frac{1}{4} \lambda_{0}$. Then $E P\left(\omega_{0}, \kappa_{0}\right)=0.29 A$ from (6) and (7), which is equivalent to reducing $|A|$ in the signal-to-noise ratio $\rho$ by a factor 0.29 . If the array gain is sufficiently large for (4) to hold, then the root-meansquare error (rmse) of $\hat{\theta}$ is increased by $343 \%$. When $\sigma_{u}=\frac{1}{10} \lambda_{0}$, then $\operatorname{EP}\left(\omega_{0}, \kappa_{0}\right)=0.82 A$ and the rmse $\hat{\theta}$ is increased by $22 \%$.

In many applications $\gamma$ and $\eta$ will be near one, but the variance of $B$ is small compared to its expected value if $M T$ is large. Suppose, for example, that $\gamma=0.99$ and $\eta=0.998, \sigma_{u}=\frac{1}{10} \lambda_{0}, M=60$, and $T=10^{5} \Delta$, where $\Delta$ is the sampling interval. Thus from (9), the standard deviation of $B$ is approximately 0.04 , as compared with $E B\left(\kappa_{0}\right)=0.82$.

Another approximation for the variance of $B$ is appropriate if $M$ or $T$ are not large but $\gamma$ and $\eta$ are near one. Suppose that $\gamma^{M} \simeq \gamma$ and $\eta^{T} \simeq \eta$. Then, from (8),

$$
\begin{aligned}
\operatorname{Var} B & \simeq \operatorname{Cov}\left\{\exp \left[i \kappa_{0} u_{0}(0)\right], \exp \left[i \kappa_{0} u_{M / 2}\left(\frac{1}{2} T\right)\right]\right\} \\
& =\exp \left(-\kappa_{0}^{2} \sigma_{u}^{2}\right)\left[\exp \left(\gamma^{M / 2} \eta^{T / 2} \kappa_{0}^{2} \sigma_{u}^{2}\right)-1\right] .
\end{aligned}
$$

This approximation will be used in the next section.

\section{SUBARRAY PROCESSING}

There is a simple method to improve the bearing estimate when the sensor spacings are perturbed by array bending. This method groups the sensors into equal-aperture adjacent subarrays. Suppose that the sensors are grouped into $R$ adjacent subarrays, each having $M_{R}=M / R$ elements, assuming for simplicity that $M$ is divisible by $R$. In addition to grouping the spatial observations, the total time sample is divided into $N$ subsamples with $T_{N}=T / N$ successive observations. A similar space-time averaging approach has been used in radio astronomy to reduce the effect of turbulence in the medium (Aime and Roddier ${ }^{19}$ ). In the acoustics literature, $\mathrm{Hahn}^{8}$ suggests processing all sensor combinations in order to estimate bearing.

Given the time and space groupings, compute the time-space Fourier transform, denoted $P_{n r}\left(\omega_{0}, \kappa\right)$, for each group. The bearing estimator is the average

$$
\bar{\theta}=\frac{1}{N R} \sum_{n=1}^{N} \sum_{r=1}^{R} \hat{\theta}_{n r},
$$

where $\hat{\theta}_{n r}$ is the bearing computed using $\left|P_{n r}\left(\omega_{0}, \kappa\right)\right|^{2}$, or by beamforming the data in the $n r$ th piece. Hinich and Rule show that a wave is resolved with probability 0.95 if the number of sensors in a linear array is approximately $8 \rho^{-1}$, where $\rho=T|A|^{2} / \sigma^{2}$ for white noise whose variance is $\sigma^{2}$. Thus, in order to achieve sufficient array gain to detect the signal,

$$
M_{R} \simeq 8(\sigma /|A|)^{2} T_{N}^{-1},
$$

provided that $T_{N}$ is sufficiently large to resolve the signal. Frequency resolution is lost due to the incoherent averaging of short-period records.

Grouping the sensors into strictly adjacent subarrays severely reduces the aperture of the subarrays, reducing the spatial resolution. For a large array, however, the aperture can be sufficiently large to obtain sufficient gain from subarrays. The idea is to trade frequency resolution for increased bearing accuracy when the array's spatial resolution is degraded due to bending.

When the signal-to-noise at each sensor is sufficiently high, the subarray aperture can be increased and the statistical improvements of sequential grouping discussed next will still hold if the end sensor in each subarray is taken from the far end of the array. For example, if $M=10$ and $R=2$, let sensor one be the first sensor in the first subarray with sensors 6-9 making up the rest. For the second array, let sensors $2-5$ be connected with sensor 10. The length of each subarray is $9 d$.

The best subarray pattern for a specific application must determined by experimentation, but the theoretical results in this paper can be used as a guide. If the space and time correlations_of the perturbations are near one, it will be shown that $\bar{\theta}$ has a smaller mse than the $\hat{\theta}$ which is computed from the entire record and the whole array.

The mean-square error for each $\hat{\theta}_{n r}$ is inversely proportional to the expected value of $\left|A B_{n r}\left(\kappa_{0}\right)\right|^{2}$. Since

$$
E|B|^{2}=\operatorname{Var} B+|E B|^{2},
$$

from (7) and (10),

$$
\begin{aligned}
E\left|B_{n r}\left(\kappa_{0}\right)\right|^{2} & =\exp \left[\left(\kappa_{0} \sigma_{u}\right)^{2}\left(\gamma^{M_{R} / 2} \eta^{T_{N} / 2}-1\right)\right] \\
& \simeq 1 \text { for } \gamma, \eta \simeq 1 .
\end{aligned}
$$

Thus when $\gamma$ and $\eta$ are near one, the expected value of the energy of the perturbation effect for each spacetime slice is approximately one. The high correlation among the sensor perturbations in each slice only produces a phase shift in the frequency-wave-number amplitude.

Suppose that the noise is white with variance $\sigma^{2}$, and 
that approximation (4) holds. From (14),

$$
\operatorname{mse}\left(\hat{\theta}_{n r}\right) \simeq \frac{3 \sigma^{2} \exp \left[\left(\kappa_{0} \sigma_{u}\right)^{2}\left(1-\gamma^{M_{R} / 2} \eta^{T_{N} / 2}\right)\right]}{2 M_{R} W^{2} T_{N}|A|^{2}(\pi \sin \theta)^{2}}
$$

for the $r$ th subarray and the $n$th time slice, assuming that the sensor pattern in the subarrays is chosen to make the subarray apertures approximate the full array aperture $W$. If the subarray lengths are only $M_{R} d$, then the aperture term in (15) is reduced by a factor of $R^{-1}$. Note that when $N=M=1, \hat{\theta}_{11}=\hat{\theta}$.

The mean-square error of the bearing estimator $\bar{\theta}$ is $(N R)^{-1} \mathrm{mse}\left(\hat{\theta}_{n r}\right)$, assuming that the noise is incoherent across the array. It then follows from (15) that

$$
\operatorname{mse}(\bar{\theta}) \simeq \frac{3 \exp \left[\left(\kappa_{0} \sigma_{u}\right)^{2}\left(1-\gamma^{M / 2 R} \eta^{T / 2 N}\right)\right]}{2 M W^{2} \rho(\pi \sin \theta)^{2}},
$$

where $\rho=T|A|^{2} / \sigma^{2}$, since $M_{R}=M / R$ and $T_{N}=T / N$.

The accuracy of $\bar{\theta}$ is superior to that of the bearing $\hat{\theta}$, which is computed from the whole array as if it were linear. From the analysis of the expected value of the perturbation factor $B$, it follows that

$$
\operatorname{mse}(\hat{\theta}) \simeq \frac{3 \exp \left(\kappa_{0} \sigma_{u}\right)^{2}}{2 M W^{2} \rho(\pi \sin \theta)^{2}} .
$$

In terms of rmse, the efficiency of $\bar{\theta}$ over $\hat{\theta}$ is

$$
\frac{\operatorname{rmse}(\bar{\theta})}{\operatorname{rmse}(\hat{\theta})} \simeq \exp \left[-\frac{1}{2}\left(\kappa_{0} \sigma_{u}\right)^{2} \gamma^{M / 2 R} \eta^{T / 2 N}\right]
$$

The upper limit of this efficiency as $\gamma \rightarrow 1$ and $\eta \rightarrow 1$ is $\exp \left[\frac{1}{2}\left(\kappa_{0} \sigma_{u}\right)^{2}\right]>1$. For example, suppose that $M=60$, $M_{R}=15, T_{N}=30, \gamma=0.99$, and $\eta=0.998$. Then if $\sigma_{u}$ $=\frac{1}{3} \lambda_{0}$,

$$
\operatorname{rmse}(\bar{\theta}) / \mathrm{rmse}(\hat{\theta})=0.139 .
$$

Even if the subarray apertures are only $\frac{1}{4} W$, the righthand side of (18) is 0.556 , i.e., the rmse using $\bar{\theta}$ is a bit over one half of that using the full array, even though the subarrays have $\frac{1}{4}$ the aperture of the full array. On the other hand, if $\sigma_{u}=\frac{1}{10} \lambda_{0}$ and the subarrays have aperture $W$, then

$$
\operatorname{rmse}(\bar{\theta}) / \operatorname{rmse}(\hat{\theta})=0.837,
$$

which means a $16 \%$ reduction in the rmse using $\bar{\theta}$. When the bending effect is small, the reduction in the aperture of some of the subarrays can wipe out the improvement in the accuracy of the bearing computed by averaging subarray bearings.

\section{THEORETICAL DESIGN TRADE-OFFS}

The expected value of the perturbation term $B\left(\kappa_{0}\right)$ is an exponentially decreasing function of the ratio $\sigma_{u} / \lambda_{0}$, which is part of the reason why the improvement in bearing accuracy due to averaging'given by expression (17) is an exponentially increasing function of $\sigma_{u} / \lambda_{0}$. If the signal has broadband energy, the accuracy improvement for a given bending parameter $\sigma_{u}$ is greatest for the smaller wavelength (higher frequency) signal components. The time slice $T_{N} \Delta$, however, must be sufficiently large to resolve the higher-frequency components. As long as the time correlation $\eta$ is near one, $T_{N}$ can be made sufficiently large to resolve the higher frequencies without a significant perturbation effect in each piece. Moreover, the smaller wavelengths require smaller apertures to achieve a given level of bearing. mse since $W$ is defined in terms of $\lambda$, and consequently $M_{R}$ can be made smaller for the higher frequencies in order to compensate for a larger $T_{N}$. It is important to note that when $W$ is very large, the mse approximation given by expression (4) is smaller than the quantization error induced by using discrete $\kappa^{\prime} s$ to maximize the wave-number spectrum (or discrete $\theta$ 's in the beamforming search method).

Intuitively, the time-space averaging method works because $T_{N}$ and $M_{R}$ are sufficiently small, compared to the variation of the array geometry, so that the perturbation effect does not attenuate the signal energy for each piece. The number of pieces should be made as large as possible, given the resolution limits.

As long as the source is stationary, the total averaging period is arbitrary. For a moving source, the time averaging can be modified to allow the bearing to track the source. For example, rather than simple averaging over time, compute the array average for each time slice,

$$
\hat{\theta}_{n}=R^{-1} \sum_{r=1}^{R} \hat{\theta}_{n r}
$$

and then fit the $\hat{\theta}_{n}$ to a polynomial track by least squares. ${ }^{20}$ In any event, the theoretical improvement over simple processing using averaging of subarrays has been demonstrated for the stochastic bending model presented in this paper, as well as for the sinusoidal patterns used in our previous paper.

\section{ACKNOWLEDGMENTS}

I wish to thank Carl Bennett (NCSL), Homer Bucker (NUC), and the referees for their helpful comments and suggestions.

*This work was supported by the Office of Naval Research under contract.

${ }^{1}$ M. J. Levin, "Least-Squares Array Processing for Signals of Unknown Form," Radio Electron. Eng. 29, 213-222 (1965).

${ }^{2}$ M. J. Hinich and P. Shaman, "Parameter Estimation for an $R$-Dimensional Plane Wave Observed with Additive Independent Gaussian Errors," Ann. Math. Stat. 43, 153-169 (1972).

${ }^{3}$ C. S. Clay, M. J. Hinich, and P. Shaman, "Error Analysis of Velocity and Direction Measurements of Plane Waves Using Thick Large-Aperture Arrays," J. Acoust. Soc. Am. 53, 1161-1166 (1973).

${ }^{4}$ F. Bryn, "Optimum Signal Processing of Three-Dimensional Arrays Operating on Gaussian Signals and Noise," J. Acoust. Soc. Am. 34, 289-297 (1962).

${ }^{5}$ J. P. Burg, "Three-Dimensional Filtering with an Array of Seismometers," Geophysics 29, 693-713 (1964).

${ }^{6}$ J. Capon, R. J. Greenfield, and R. J. Kolker, “Multidimensional Maximum-likelihood Processing of a Large Aperture Seismic Array," Proc. IEEE 55, 192-211 (1967).

${ }^{7}$ W. S. Liggett, Jr., "Passive Sonar Processing for Noise with Unknown Covariance Structure," J. Acoust. Soc. Am. 51, 24-30 (1972).

${ }^{8}$ W. R. Hahn, "Optimum Signal Processing for Passive Sonar Range and Bearing Estimation," J. Acoust. Soc. Am. 58, 
201-207 (1975).

${ }^{9}$ G. C. Carter and C. H. Knapp, "Time Delay Estimation," in Proceedings of the 1976 IEEE Conference on Acoustics, Speech and Signal Processing (IEEE, New York, 1976), pp. 357-360.

${ }^{10} \mathrm{~J}$. Capon and P. Green, "Statistical Accuracy of Data Used in Seismic Inversion," Geophys. J. R. Astron. Soc. 21, 373-386 (1970).

${ }^{11}$ V. H. MacDonald and P. J. Schultheiss, "Optimum Passive Bearing Estimation in a Spatially Incoherent Noise Environment," J. Acoust. Soc. Am. 46, 37-43 (1969).

${ }^{12} \mathrm{~A}$. M. Walker, "On the Estimation of a Harmonic Component in a Time Series with Stationary Independent Residuals," Biometrika 58, 21-36 (1971).

${ }^{13} \mathrm{C}$. R. Rao, Linear Statistical Inference and Its Applications (Wiley, New York, 1965).

${ }^{14}$ N. F. Barber, "Design of 'Optimum' Arrays for DirectionFinding," Electron. Radio Eng. 36, 222-232 (1959).
${ }^{15}$ The bearing is aliased if $|\cos \theta|>\lambda_{0} / 2 d$. Suppose that $\lambda_{0} /$ $2 d<\cos \theta<3 \lambda_{0} / 2 d$. Then the source will be detected at the alias $\theta_{a}$, where $\cos \theta_{a}=\cos \theta-\lambda_{0} / d$, e.g., if $\theta=60^{\circ}$ and $d=\lambda_{0}$, then $\theta_{a}=120^{\circ}$.

${ }^{16} \mathrm{C}$. S. Clay and M. J. Hinich, "Use of a Two-Dimensional Array to Receive an Unknown Signal in a Dispersive Waveguide," J. Acoust. Soc. Am. 47, 435-440 (1970).

${ }^{17}$ M. J. Hinich and W. Rule, "Bearing Estimation Using a Large Towed Array," J. Acoust. Soc. Am. 58, 1023-1029 (1975).

${ }^{18} \mathrm{~L}$. S. Cahoon and M. J. Hinich, "A Method for Locating Targets using Range Only," IEEE Trans. Inf. Theory IT-22 (2), 217-225 (1976).

${ }^{19} \mathrm{C}$. Aime and F. Roddier, "Imaging through Turbulence with Telescope Arrays," Opt. Commun. 19, 57-60 (1976).

${ }^{20}$ J. U. Farley, M. J. Hinich, and T. W. McGuire, "Testing for a Shift in the Slopes of a Multivariate Linear Time Series Model," J. Econometrics 3, 297-318 (1975). 